\title{
RAZONAMIENTO Y ARGUMENTACIÓN EN CIENCIAS. DIFERENTES PUNTOS DE VISTA EN EL CURRÍCULO OFICIAL
}

\author{
GARCÍA DE CAJÉN, SILVIA, ${ }^{1}$ DOMÍNGUEZ CASTIÑEIRAS, JOSÉ MANUEL, ${ }^{2}$ GARCÍA- \\ RODEJA FERNÁNDEZ, EUGENIO' \\ ${ }^{1}$ Departamento de Profesorado de Física y Química. UN del Centro. Olavarría. Argentina \\ sgarcia@fio.unicen.edu.ar \\ 2 Departamento de Didáctica das Ciencias Experimentais. Universidad de Santiago de Compostela \\ ddacabdz@usc.es
}

\begin{abstract}
Resumen. Las perspectivas constructivistas del aprendizaje y de la enseñanza de las ciencias señalan la importancia de que los alumnos aprendan a razonar y a argumentar. Los nuevos currículos de ciencias deberían tener en cuenta el campo de las destrezas cognitivas y de pensamiento. A ese respecto, hemos diseñado un marco referencial y una clave de vaciado y de categorización de contenidos procedimentales que nos han permitido evaluar si el currículo oficial de la Argentina fomenta el aprendizaje de las estrategias de razonamiento y de argumentación en niveles de enseñanza no universitarios y en la formación de profesores.

Palabras clave: Currículo, enseñanza de las ciencias, procedimientos, razonamiento, argumentación.

Summary. The constructivist approaches to learning in science teaching show towards the importance of students learning how to reason an argument. The new science curriculum should contemplate the field of cognitive and thinking skills. To evaluate if the official curriculum in Argentina promotes reasoning and argumenting strategies at different educational levels and in teacher education, a reference scheme and a procedure for categorizing methodological content has been designed. Keywords. Curriculum, science teaching, skills, reasoning, argumentation.
\end{abstract}

\section{INTRODUCCIÓN. SITUACIÓN DEL PRO- BLEMA}

La investigación ha puesto de manifiesto la diferente naturaleza del conocimiento declarativo (saber decir) y el procedimental (saber hacer). Ambos tipos de conocimiento se adquieren por procesos diferentes y hasta cierto punto independientes (Anderson, 1983; Pozo, 1996). Algunos autores (Pozo y Gómez, 1998) señalan la dificultad que supone para el alumnado convertir sus conocimientos científicos descriptivos y conceptuales en acciones o predicciones eficaces y viceversa. Millar y Driver (1987) advierten sobre la artificialidad de diferenciar entre conceptos y procesos, señalando que aprender conceptos implica procesos y el aprendizaje de éstos se realiza siempre sobre conceptos concretos.

Latour y Woolgar (1995) y Driver y Newton (2000) ponen de manifiesto una visión de la ciencia que tiene claras implicaciones para la enseñanza: consideran la importancia de la observación y de la experimentación pero, además, ponen énfasis en el razonamiento deductivo y en el enfoque como proceso de construcción social del conocimiento. Desde esta perspectiva, el razonamiento se manifiesta fundamental para el aprendizaje de las ciencias (Kuhn, 1993; Jiménez, 1998).

A este respecto, Duschl (1995) propone que, en un ambiente de aprendizaje de ciencias, deberá construirse conjuntamente el campo del conocimiento epistemológico y científico, el campo de las destrezas cognitivas y de pensamiento y el campo de las destrezas sociales y de comunicación, lo que será necesario tener en cuenta al evaluar el aprendizaje (Domínguez, 2000).

Los contenidos procedimientales, igual que los conceptuales, deben enseñarse (Pro, 1995) y, para conseguir su aprendizaje, deben acompañarse de cambios en las acti- 
tudes y valores (Duschl, 1995). Sin embargo, con frecuencia, el profesorado supone que el alumnado desarrollará de forma natural los procedimientos que faciliten el aprendizaje (Duschl, 1990). El conocimiento procedimental, el uso de estrategias de razonamiento, contribuye positivamente al éxito académico (Duschl, 1995; Domínguez, 2000).

Forma parte de nuestra experiencia profesional compartir espacios institucionales, de formación y capacitación, con el profesorado de ciencias. Parece que éste, a través de una visión piagetiana, liga la evolución del razonamiento al desarrollo biológico, mientras que desde la comunidad científica se sostiene que el progreso en la capacidad de razonamiento viene de la propia experiencia en un contexto educativo apropiado. Desde este punto de vista, Van Hiele (1986), Eichinger y otros (1991), Pontecorvo y Girardet (1993) y Kelly y otros (1996) proponen hacer evolucionar la idea de conocimiento específico de una materia, desde el conocimiento proposicional a la capacidad de justificación. La enseñanza de las ciencias, además de el qué de las ciencias, pretende que se participe en su forma de trabajar (Jiménez et al., 1998). Esto implica que, además de la construcción de modelos sobre el mundo físico y natural, es necesario utilizarlos en la interpretación de los hechos para dotarlos de funcionalidad en el desarrollo de la capacidad de razonar sobre cuestiones de ciencias (Domínguez, 2000).

Las reformas educativas en diferentes países parecen coincidir en que uno de los fines principales de la enseñanza de las ciencias en la etapa obligatoria es la alfabetización científica, relacionada con la formación de ciudadanos responsables que posean capacidad crítica a la hora de tomar decisiones. En tiempos de reforma educativa resulta de interés conocer si el marco legal incluye las destrezas de razonamiento y de argumentación (Jiménez et al., 1998).

En la Argentina, el marco referencial de los saberes educativos viene dado por los contenidos básicos comunes (CBC) acordados a nivel nacional (MCE, 1995, 1997a, 1997b, 1998). Estos CBC son utilizados para la reformulación de los diseños curriculares de cada jurisdicción o provincia. Posteriormente el centro escolar debería elaborar el proyecto institucional, y el profesorado, sus proyectos de aula, tomando como base los documentos provinciales y nacionales.

Los CBC del Área de Ciencias Naturales de EGB y Polimodal (MCE, 1995, 1997a) se organizan en 6 bloques. Cuatro corresponden a contenidos conceptuales (B1: La vida y sus propiedades; B2: El mundo físico; B3: Estructura y cambios de la materia y $B 4$ : La Tierra y sus cambios); uno, a contenidos procedimentales (B5: Procedimientos en la investigación escolar del mundo natural); y otro, a contenidos actitudinales (B6: Actitudes generales relacionadas con el mundo y con las ciencias naturales). Los CBC de formación de profesores (MCE, 1997b, 1998) también clasifican los contenidos en conceptuales, procedimentales y actitudinales.
Este estudio, que es parte de un proyecto más amplio en el que se investiga además la argumentación del profesorado y la que aparece en los libros de texto, tiene por objetivo poner en evidencia si se fomenta el aprendizaje de estrategias de razonamiento y de argumentación en los niveles de educación general básica (EGB), de educación polimodal (POL) y en formación de profesores de ciencias (FPC)

Según las fuentes indicadas, el nuevo currículo es un constructo de discursos pedagógicos y disciplinares. Especialistas de las disciplinas han redactado los documentos B1-4, y especialistas de la pedagogía, los B5-6, por lo que es posible que distintas partes del currículo otorguen diferente énfasis a las estrategias investigadas.

Centramos la investigación en los contenidos procedimentales de los siguientes bloques del currículo:

a) B5, contenidos procedimentales no relacionados con los contenidos conceptuales;

b) B1-4, contenidos procedimentales relacionados con los contenidos conceptuales del área.

\section{ESTRATEGIA DE RECOGIDA DE INFOR- MACION Y SU TRATAMIENTO}

\section{La muestra}

Constituyen la muestra investigada, los contenidos procedimentales presentes en los documentos oficiales (DO) enviados por el Ministerio de Cultura y Educación de la Nación (MCE), y por la Dirección General de Cultura y Educación de la Provincia de Buenos Aires (DGCE) a las instituciones escolares, en el marco de la reforma educativa (Tabla I).

La recogida de la documentación se realizó en bibliotecas de centros escolares de Olavarría (Argentina). Los documentos disponibles para la consulta, a cinco años de implementada la reforma, corresponden a los siguientes niveles educativos: EGB, POL y FP de EGB y POL.

Tabla I

\begin{tabular}{|ll|l|l|}
\hline $\begin{array}{l}\text { Nivel de } \\
\text { enseñanza }\end{array}$ & DO & Ámbito \\
\hline EGB & 6-14 años & $\begin{array}{l}\text { MCE (1995) } \\
\text { DGCE (1995) } \\
\text { DGCE (1999a) }\end{array}$ & $\begin{array}{l}\text { nacional } \\
\text { nacional } \\
\text { provincial } \\
\text { provincial }\end{array}$ \\
\hline POL & 15-18 años & MCE (1997a) & nacional \\
\hline FPC & duración 4 años & $\begin{array}{l}\text { MCE (1997b) } \\
\text { MCE (1998) } \\
\text { DGCE (1999b) }\end{array}$ & $\begin{array}{l}\text { nacional } \\
\text { nacional } \\
\text { provincial }\end{array}$ \\
\hline
\end{tabular}


Señalamos que el diseño curricular provincial del nivel EGB (DGCE, 1999a) se ha obtenido por gestiones ante autoridades provinciales.

La formación de profesores se realiza en instituciones de educación superior: facultades e institutos terciarios (no integrados en la universidad).

\section{El marco interpretativo de los contenidos procedi- mentales del currículo de ciencias}

Con el fin de evitar posibles sesgos en la interpretación de los contenidos investigados, hemos consultado la literatura científica relacionada con los campos de la psicología, de la filosofía y de la investigación educativa, sobre el significado de los términos estrategia, razonamiento y argumentación.

\section{Estrategias en la literatura científica}

De los diferentes significados dados al término estrategias en la literatura científica especializada (Monereo y Castelló, 1997; Pozo et al., 1995; Sevilla, 1994; Tishman et al., 1994), consideramos que éstas comprenden, además del plan de acción, la propia acción y su valoración (Monereo y Castelló, 1997) y requieren de la utilización de destrezas (Pozo et al., 1995). En consecuencia, necesitamos un marco de referencia que nos permita identificar las destrezas utilizadas, presentes en las estrategias de razonamiento y de argumentación.

\section{Razonamiento y argumentación}

Durante mucho tiempo se asoció el razonamiento científico a la práctica del razonamiento inferencial (Vergnaud, 1994), entendido éste como la capacidad del individuo para plantearse preguntas y hablar consigo mismo, cuando resuelve problemas, para así comprobar interiormente sus planteamientos hipotéticos y tomar decisiones razonadas. Los procesos inductivos parecen destinados a la formulación de hipótesis, de las que se pueden extraer consecuencias deductivas (Sevilla, 1994).

El significado de razonamiento ha cambiado en las últimas décadas. Desde la nueva filosofía de la ciencia, se sostiene que la construcción del conocimiento científico no responde a patrones de razonamiento inferencial, sino que se construye como un razonamiento que se exterioriza ante una comunidad científica que lo discute y lo evalúa. Giere (1994) considera que el razonamiento en ciencias se relaciona con los procesos de toma de decisiones que se practican cuando hay que elegir entre distintas hipótesis. El mismo autor fundamenta el abandono del razonamiento como lógica inductivista y asume el nuevo enfoque del mismo como un razonamiento práctico, en términos de modelos y decisiones basadas en los datos (Giere, 1999).

Respecto del concepto de argumentación también existen diversos significados. Driver y Newton (2000) diferencian la lógica -disciplina académica que presenta reglas aisladas para la producción de deducciones co- rrectas a partir de premisas dadas- de cómo la gente razona en realidad. La argumentación puede considerarse como una actividad individual, a través de la reflexión y de la escritura, o como una actividad social que tiene lugar dentro de un grupo. Cuando un alumno habla ciencia en un contexto social, recibe la influencia de la presencia de sus compañeros (Vergnaud, 1994), da lugar a un tipo de razonamiento colectivo o compartido relacionado con la argumentación social (Pontecorvo y Girardet, 1993).

Independientemente del carácter individual o colectivo, Driver y Newton (2000), definen argumentación como el proceso por el que se da una razón a favor o en contra de una proposición o línea de acción; esta interpretación de argumentación la denomina Kuhn (1993) retórica y Driver y Newton (2000), didáctica. Ejemplos de este tipo de argumentación son comunes en las clases de ciencias, en las que el profesor proporciona una explicación a un grupo de estudiantes con la intención de ayudarles a verla razonable. Esta argumentación unilateral tiene sus limitaciones en situaciones de enseñanza, desde la perspectiva de la construcción social de la ciencia, pues son los estudiantes los que han de desarrollar por sí mismos estas destrezas de argumentación.

Enlazando con lo anterior surge la definición de argumentación dialógica o de voz múltiple (Driver y Newton, 2000), que tiene lugar cuando se examinan, por un individuo o en el seno de un grupo, diferentes perspectivas para llegar a un acuerdo sobre cuáles son las afirmaciones del conocimiento que se aceptan o las líneas de acción que se toman en consideración. Este tipo de argumentación surge en las clases de ciencias cuando se da a los estudiantes la oportunidad de abordar un problema en pequeño grupo o cuando, en situación de discusión en la clase, el profesor dirige un debate para identificar diferentes líneas de pensamiento e invita a los estudiantes a evaluarlas con el fin de ir hacia un resultado con el que todo el mundo esté de acuerdo. A este respecto, Kuhn (1993) identifica discusión o controversia (argument) con argumentación.

Se infiere de lo dicho, la importancia de aprender a hablar ciencia (Lemke, 1993) y de la necesidad de organizar las aulas de manera que los estudiantes tengan voz y darles así oportunidades para que practiquen el razonamiento y la argumentación. Estas oportunidades les proporcionarán la importante tarea de elaborar argumentos razonados, individual y colectivamente, para que, de esta manera, también desarrollen, ellos mismos, este tipo de destrezas (Domínguez, 2000).

Consideramos que, para que surja la argumentación, es determinante la existencia de un contexto de discusión o controversia entre alumnos, y entre alumnos y profesores de ciencias. Se considera necesario crear ambientes de aprendizaje que demanden al alumnado la resolución de problemas auténticos (Jiménez, 1998; Kelly et al., 1996). El propio planteamiento del problema es un aspecto fundamental en la construcción del conocimiento que se debería incluir en el referencial, si bien este aspecto no aparece en la literatura consultada, en la que 
Tabla II

\begin{tabular}{|c|c|c|}
\hline Estrategias & Destrezas implicadas & CD \\
\hline $\begin{array}{l}\text { Razonamiento inductivo } \\
\text { MCE }(1997 \mathrm{c})\end{array}$ & $\begin{array}{l}\text {-Análisis de casos particulares de fenómenos naturales } \\
\text {-Análisis y control de variables } \\
\text {-Comparar y establecer relaciones } \\
\text {-Buscar regularidades } \\
\text {-Anticipar resultados } \\
\text {-Formular generalizaciones } \\
\text {-Elaborar conjeturas } \\
\text {-Formular hipótesis }\end{array}$ & $\begin{array}{l}\text { I. } 1 \\
\text { I. } 2 \\
\text { I. } \\
\text { I. } \\
\text { I. } \\
\text { I. } \\
\text { I. } \\
\text { I. } \\
\text { I }\end{array}$ \\
\hline $\begin{array}{l}\text { Razonamiento deductivo } \\
\text { MCE }(1997 \mathrm{c})\end{array}$ & $\begin{array}{l}\text {-Comprobar hipótesis en casos particulares } \\
\text {-Predicción de fenómenos o resultados a partir de modelos }\end{array}$ & $\begin{array}{l}\text { D. } 1 \\
\text { D. } 2\end{array}$ \\
\hline $\begin{array}{l}\text { Argumentación } \\
\text { Duschl, } 1990 \\
\text { Kuhn, 1993 } \\
\text { Pontecorvo y Girardet, } 1993 \\
\text { Jorba y Sanmartí, } 1996 \\
\text { Jiménez, 1998 } \\
\text { Driver y Newton, } 1997 \\
\text { Sanmartí, 1997 } \\
\text { Jiménez et al., 1997, } 1998 \\
\text { Sardá y Sanmartí, } 2000 \\
\text { Izquierdo, 2000 }\end{array}$ & $\begin{array}{l}\text {-Identificar pruebas y datos } \\
\text {-Diferenciar hechos y explicaciones teóricas } \\
\text {-Comparar modelos teóricos y situación física real } \\
\text {-Identificar razones } \\
\text {-Elaborar razones } \\
\text {-Elaborar explicaciones } \\
\text {-Formular conclusiones } \\
\text {-Evaluar una hipótesis o enunciado } \\
\text {-Justificar respuestas } \\
\text {-Analizar críticamente } \\
\text {-Realizar crítica } \\
\text {-Elaborar, modificar y justificar hipótesis sobre fenómenos naturales } \\
\text {-Dar argumentos y contraargumentos } \\
\text {-Evaluar consistencia y cohesión de la argumentación } \\
\text {-Usar lenguaje de la ciencia } \\
\text {-Resolver un conflicto mediante negociación social } \\
\text {-Evaluar alternativas } \\
\text {-Reflexionar acerca de la evidencia } \\
\text {-Evaluar la viabilidad de conclusiones científicas } \\
\text {-Buscar coherencia y globalidad }\end{array}$ & $\begin{array}{l}\text { A. } 1 \\
\text { A. } \\
\text { A. } \\
\text { A. } \\
\text { A. } \\
\text { A. } \\
\text { A. } \\
\text { A. } \\
\text { A. } \\
\text { A. } \\
\text { A. } 11 \\
\text { A. } 12 \\
\text { A. } 13 \\
\text { A. } 14 \\
\text { A. } 15 \\
\text { A. } 16 \\
\text { A. } 17 \\
\text { A. } 18 \\
\text { A. } 19 \\
\text { A. } 20\end{array}$ \\
\hline
\end{tabular}

se parte de un problema ya planteado. Queda, pues, abierto el interrogante: ¿Qué vinculación establece la literatura entre las estrategias de razonamiento y de argumentación y el planteamiento de problemas?

En un estudio interpretativo como el presente, es necesario establecer criterios (Kesidou y Duit, 1993) que permitan evaluar determinados contenidos procedimentales del currículo investigado, tales como estrategias de razonamiento, conceptualizado como razonamiento inferencial, y de argumentación, conceptualizada como argumentación dialógica. Dichos criterios nos permitirán elaborar un marco referencial que cumplirá una función orientadora en el proceso de análisis y categorización de los contenidos procedimentales.

Marco referencial para la categorización de las estrategias de razonamiento y de argumentación en la ciencia escolar

No hemos encontrado en la literatura científica una clasificación que oriente la identificación de estrategias de razonamiento y de argumentación, pero sí es posible, a partir de dicha literatura, hacer explícitos los criterios (Tabla II) que permiten clasificar determinados procedimientos del currículo oficial (Tablas V y VIII) en categorías de razonamiento y de argumentación (Tablas IX y X). La elección de las categorías razonamiento induc- tivo, razonamiento deductivo y argumentación (Tabla II) se realiza considerando que durante mucho tiempo la concepción predominante sobre el saber científico estuvo asociada a la realización de inferencias inductivas y deductivas, mientras que la concepción actual lo considera como un saber argumentado y construido colectivamente (Vergnaud, 1994; Driver y Newton, 2000).

La presentación de tres categorías de estrategias no implica que las consideremos como clases excluyentes. Por el contrario, opinamos que la argumentación, en su carácter de estrategia cognitivo-lingüística (Jorba y Sanmartí, 1996), es una estrategia compleja que requiere de recursos cognitivos, por ejemplo, destrezas de razonamiento inferencial, que faciliten la reflexión y el metaconocimiento (Pozo et al., 1995; Pozo, 1996; Pozo y Gómez, 1998). La investigación educativa pone énfasis en que los recursos metacognitivos (Minick y Albermann, 1994) deberían ser aprendidos estratégicamente y valora su aprendizaje como una experiencia educativa en sí misma (Head, 1985; Claxton, 1991; Campanario y Otero, 2000).

Hemos elaborado el marco referencial (Tabla II) consultando, en primer lugar, los documentos de la muestra investigada (Tabla I) pero, dado que éstos no hacen explícito qué se entiende por razonamiento y argumentación, se amplió la consulta a otros documentos oficia- 
les (DGCE, 1998; MCE, 1997c) y encontró información sobre razonamiento inductivo y deductivo. Ya que ninguno de los documentos anteriores indica qué se entiende por argumentación en ciencias, consultamos la literatura especializada (Duschl, 1990; Kuhn, 1993; Pontecorvo y Girardet, 1993; Jorba y Sanmartí, 1996; Jiménez et al., 1997, 1998; Sanmartí, 1997; Jiménez, 1998; Sardá y Sanmartí, 2000; Izquierdo, 2000; Driver y Newton, 2000) y, a partir de la misma, construimos las categorías de estrategias de argumentación. En dicho marco identificamos cada destreza con un código, indicando con una letra (I, D, A) las categorías (inductivo, deductivo, argumentativo) y con un número, el orden, sin implicar esto ningún tipo de secuenciación.

Las destrezas encontradas en la literatura (Tabla II) pueden dar lugar a confusión, por ejemplo, formular hipótesis y elaborar hipótesis. Según Minick y Albermann (1994), la elaboración, como tarea académica, supone la búsqueda en la memoria de conocimiento declarativo y procedimientos relevantes $\mathrm{y}$, posteriormente, la formulación de un plan para realizar la tarea, en este caso, la hipótesis. Si ésta se elabora como parte de un proceso de elección entre hipótesis que compiten (Giere, 1994), es posible considerar la destreza elaborar hipótesis en la categoría de argumentación. En cambio, la literatura que cita la destreza formular hipótesis, lo hace desde un enfoque proposicional del razonamiento.

Señalamos también que en una misma categoría se enuncian destrezas que no son excluyentes entre sí, como, por ejemplo, las destrezas elaborar razones (A.5) y elaborar explicaciones (A.6). Sardá y Sanmartí (2000) relacionan la capacidad de seleccionar evidencias significativas en los modelos de la ciencia con la destreza de elaborar razones. Sanmartí (1997) considera que la explicación científica tiene como objetivo la comprensión desde la ciencia de referencia. Señala, Izquierdo (2000), que elaborar explicaciones implica utilizar el patrón temático sobre el género textual explicativo, relacionado con determinadas destrezas cognitivolingüísticas.
Frente a las destrezas de razonamiento resultan numerosas las destrezas de argumentación. Quizás se deba a diferentes conceptualizaciones de la argumentación en la investigación educativa o bien a la complejidad que implica la argumentación en ciencias.

\section{Proceso de extracción de contenidos procedimentales}

Bloque 5. Procedimientos en la investigación escolar del mundo natural

- Vaciado de propósitos

Señala el DGCE (1999a, p. 32) que el aprendizaje de los procedimientos debe realizarse con diversos grados de profundidad, de acuerdo con la complejidad conceptual de los problemas en estudio. El currículo de educación polimodal incorpora los contenidos procedimentales con el propósito de que el alumnado aprenda a tomar decisiones en base a argumentos y a utilizar modelos (Tabla III). Dichos contenidos contribuyen al desarrollo de capacidades importantes para el trabajo de las ciencias, que pueden enriquecer la participación y el desempeño de las personas en la vida cotidiana.

En la FPC es propósito (MCE, 1998) que los profesores no sólo sepan utilizar, sino también analizar, los princi-

\section{Tabla III}

\begin{tabular}{|l|l|}
\hline DO & \multicolumn{1}{c|}{ Propósitos } \\
\hline \multirow{2}{*}{ MCE, (1997a) } & $\begin{array}{l}\text {-Planificar, desarrollar y analizar distintos } \\
\text { diseños de investigación que impliquen } \\
\text { control de variables y tomar decisiones en } \\
\text { base a argumentos o resultados } \\
\text { experimentales. } \\
\text {-Utilizar modelos para predecir fenómenos } \\
\text { o resultados, y para elaborar y analizar } \\
\text { conclusiones de investigaciones. }\end{array}$ \\
\hline
\end{tabular}

Tabla IV

\begin{tabular}{|c|c|c|c|c|}
\hline \multirow[t]{2}{*}{$\begin{array}{l}\text { Categorías de contenidos en el bloque } 5 \\
\text { Procedimientos en la investigación escolar } \\
\text { del mundo natural }\end{array}$} & \multirow[t]{2}{*}{$\begin{array}{l}\text { Código } \\
\text { de la } \\
\text { categoría }\end{array}$} & \multicolumn{3}{|c|}{$\begin{array}{l}\text { Distribución de contenidos } \\
\text { procedimentales } \\
\text { en el bloque } 5 \text { de cada nivel }\end{array}$} \\
\hline & & EGB* & POL & PCS \\
\hline Formulación de preguntas y explicaciones provisorias & $\mathrm{C} 1$ & $3 / 3 / 2$ & 2 & 2 \\
\hline Selección, recolección y organización de información & $\mathrm{C} 2$ & $6 / 9 / 9$ & 2 & 2 \\
\hline Interpretación de la información & $\mathrm{C} 3$ & $3 / 3 / 3$ & 2 & 4 \\
\hline Diseño de investigaciones & $\mathrm{C} 4$ & $1 / 3 / 2$ & 1 & 1 \\
\hline Comunicación & $\mathrm{C} 5$ & $2 / 4 / 2$ & 2 & 2 \\
\hline
\end{tabular}


pales procedimientos generales involucrados en los procesos de investigación; por ejemplo, el razonamiento y la argumentación.

\section{- Organización de los contenidos procedimentales}

En el bloque 5, los especialistas curriculares organizan los contenidos en cinco categorías (Tabla IV). Indicamos cuántos contenidos de cada categoría son enunciados en cada nivel de enseñanza, pero, dada la extensión del nivel de EGB*, en éste, lo hacemos por ciclos $\left(1^{\mathrm{o}}, 2^{\mathrm{o}}, 3^{\mathrm{o}}\right)$.

El currículo oficial propone el aprendizaje de los contenidos procedimentales desde el primer ciclo de EGB, siendo en este nivel en el que los especialistas curriculares enuncian el mayor número de contenidos procedimentales.

Parte de este trabajo consiste en establecer la existencia de relaciones entre los procedimientos investigados (Tablas V y VIII) y el patrón de argumentación, tornándose fundamental encontrar un indicador que permita reconocer qué contenidos procedimentales del currículo implican la utilización del patrón argumentativo. Teniendo en cuenta que la argumentación (Pontecorvo y Girardet, 1993; Driver y Newton, 2000) está relacionada con actividades colectivas, consideramos el campo social y de comunicación (Duschl, 1995) como condicionante de la práctica de la argumentación.
Decidimos centrar en la categoría Comunicación la búsqueda de evidencia del rol que tiene el componente social en la construcción de la ciencia escolar. En los fundamentos de esta categoría, el marco legal (MEC, 1995 , p. 140) sostiene que «el proceso de producción de conocimientos científicos es colectivo, requiere de la colaboración de los grupos científicos donde la comunicación posibilita el intercambio entre ellos y viabiliza la construcción de un conocimiento objetivo». Señálase también que la comunicación no se circunscribe a la comunicación de resultados, sino que abarca todo el proceso de construcción de conocimientos. Esta perspectiva de la comunicación es propicia para la práctica de la argumentación en las clases de ciencias.

- Categorías del bloque 5, que incluye las estrategias de razonamiento y argumentación

La literatura (Pro, 1998) aporta formas de clasificar los contenidos procedimentales. En la organización curricular de procedimientos propuesta por Pozo y otros, (1995) se incluyen las estrategias de razonamiento en la categoría Análisis de la información y realización de inferencias. En algunos currículos se consideran la interpretación y el análisis como dos categorías distintas (Pozo et al., 1995; Pro, 1998). En cambio, Sevilla (1994) señala que la interpretación requiere del proceso de análisis, criterio que coincide con la categorización del currículo oficial investigado (Tabla IV), ya que encontramos los procedimientos de análisis, la formulación de

Tabla V

\begin{tabular}{|c|c|c|c|}
\hline $\begin{array}{l}\text { Nivel de } \\
\text { enseñanza }\end{array}$ & DO & $\begin{array}{l}\text { Transcripción de contenidos procedimentales enunciados en la categoría } \\
\text { Interpretación de información del bloque } 5 \text { del área de ciencias naturales }\end{array}$ & CD \\
\hline EGB1 & $\begin{array}{l}\text { MCE, } 1995, \\
\text { p. } 164\end{array}$ & $\begin{array}{l}\text {-Reconocimiento de diferencias entre objetos o hechos semejantes } \\
\text {-Reconocimiento de semejanzas entre objetos o hechos diferentes } \\
\text {-Predicción de comportamientos en base a resultados de exploraciones realizadas }\end{array}$ & $\begin{array}{l}1 . \mathrm{a} \\
1 . \mathrm{b} \\
1 . \mathrm{c}\end{array}$ \\
\hline EGB2 & $\begin{array}{l}\text { MCE, } 1995, \\
\text { p. } 165\end{array}$ & $\begin{array}{l}\text {-Integración de diversos aspectos de la información } \\
\text { (procedentes de observaciones directas o de fuentes secundarias) } \\
\text { e inferir algo de ellos } \\
\text {-Uso de pautas o relaciones de la información, medidas u observaciones } \\
\text { para hacer predicciones } \\
\text {-Identificación de tendencias o relaciones en la información }\end{array}$ & $\begin{array}{l}2 . \mathrm{a} \\
2 . \mathrm{b} \\
2 . \mathrm{c}\end{array}$ \\
\hline EGB3 & $\begin{array}{l}\text { MCE, } 1995, \\
\text { p. } 165\end{array}$ & $\begin{array}{l}\text {-Diferenciación de conclusiones que se ajustan a evidencias de inferencias } \\
\text { que van más allá de las evidencias } \\
\text {-Predicción de comportamientos a partir del análisis de gráficos y de procesos } \\
\text {-Elaboración de modelos y análisis de la pertinencia de los mismos }\end{array}$ & $\begin{array}{l}3 . a \\
3 . b \\
3 . c\end{array}$ \\
\hline Polimodal & $\begin{array}{l}\text { MCE, 1997a, } \\
\text { p. } 129\end{array}$ & $\begin{array}{l}\text {-Análisis e interpretación de situaciones a partir de principios o modelos } \\
\text {-Evaluación de la pertinencia de procesos, materiales o aparatos a emplear } \\
\text { en la investigación }\end{array}$ & $\begin{array}{l}4 . \mathrm{a} \\
4 . \mathrm{b}\end{array}$ \\
\hline $\begin{array}{l}\text { Formación } \\
\text { de profesores } \\
\text { de ciencias }\end{array}$ & $\begin{array}{l}\text { MCE, } 1998, \\
\text { p. } 26 ; 1997 b, \\
\text { p. } 150 \\
\text { DGCE, } 1999 b \text {, } \\
\text { p. } 33 .\end{array}$ & $\begin{array}{l}\text {-Análisis e interpretación de situaciones a partir de principios o modelos } \\
\text {-Evaluación de la pertinencia de procesos, materiales o } \\
\text { aparatos a emplear en la investigación } \\
\text {-Comparación de diferentes teorías y modelos } \\
\text {-Análisis crítico y contextualizado de cada teoría o modelo en un } \\
\text { marco sociocultural }\end{array}$ & $\begin{array}{l}\text { 5.a } \\
3 . \mathrm{b} \\
\text { 5.c } \\
\text { 5.d }\end{array}$ \\
\hline
\end{tabular}


predicciones y la realización de inferencias, incluidos en la categoría Interpretación de la información. Decidimos buscar, en esta categoría, las estrategias de razonamiento mientras que, para determinar las de argumentación, hemos relacionado los procedimientos incluidos en aquella categoría con los de Comunicación.

El vaciado de la información (TablaV) se realiza tomando como clave la adaptada de Couce y otros (1998).

\section{Bloques disciplinares}

En los CBC de EGB (MCE, 1995) cada bloque disciplinar presenta relaciones entre contenidos conceptuales y contenidos procedimentales específicos (p.e., B2 de

Tabla VI

\begin{tabular}{|l|l|}
\hline $\begin{array}{l}\text { Transcripción de contenidos } \\
\text { procedimentales del núcleo temático } \\
\text { Electricidad y magnetismo } \\
\text { del B2 de EGB3 }\end{array}$ & Categoría \\
\hline $\begin{array}{l}\text { Diseño, construcción y análisis del } \\
\text { funcionamiento de aparatos simples } \\
\text { (timbres, parlantes) }\end{array}$ & $\mathrm{C} 4, \mathrm{C} 3$ \\
\hline $\begin{array}{l}\text { Diseño y ejecución de exploraciones } \\
\text { de efectos de campos magnéticos variables } \\
\text { sobre circuitos eléctricos (leyes de inducción) }\end{array}$ & $\mathrm{C} 4, \mathrm{C} 3$ \\
\hline $\begin{array}{l}\text { Observación y análisis del funcionamiento } \\
\text { de transformadores, generadores y motores }\end{array}$ & $\mathrm{C} 2, \mathrm{C} 3$ \\
\hline
\end{tabular}

EGB2: Electricidad y magnetismo, contenido conceptual Circuitos eléctricos; procedimental Diseño, construcción y análisis de funcionamiento de circuitos simples). En los CBC de polimodal (MCE, 1997a), la redacción de procedimientos no es tan detallada. En los CBC de formación de profesores se encuentran solamente contenidos conceptuales.

- Contenidos procedimentales de los bloques disciplinares y su identificación con las categorías del bloque 5

Clasificamos todos los procedimientos según las cinco categorías indicadas en el bloque 5 (Tabla IV). Con frecuencia encontramos que están compuestos por más de un procedimiento y que pueden corresponder o no a una misma categoría del bloque 5. A modo de ejemplo (Tabla VI) transcribimos procedimientos extraídos de la temática Electricidad y magnetismo del bloque 2 (Mundo físico) de EGB3 (MCE, 1995).

Del proceso de categorización anterior, surge la siguiente distribución de los contenidos por disciplina y categoría procedimental (Tabla VII). En el nivel de EGB, indicamos la distribución por ciclo $\left(1^{\circ}, 2^{\circ}, 3^{\circ}\right)$.

Se pone de manifiesto la escasa (B2 y B4, en EGB2 y EGB3), y en algunos caso nula (POL), presencia de la categoría comunicación en los contenidos procedimentales de los bloques disciplinares.

De la lectura de cada contenido (MCE, 1995, p. 140) se infiere que la comunicación, en los bloques disciplinares, sólo implica comunicación de resultados o de información, contrariamente a lo que el mismo documento sostiene respecto a que la comunicación no se circunscribe a la comunicación de resultados, sino que atraviesa todo el proceso de construcción de conocimientos.

\begin{tabular}{|c|c|c|c|c|c|c|c|c|c|}
\hline \multirow{3}{*}{$\begin{array}{l}\text { Categorías de contenidos en el bloque } 5 \\
\text { Procedimientos en la investigación escolar } \\
\text { del mundo natural }\end{array}$} & \multirow{3}{*}{$\begin{array}{c}\text { Código } \\
\text { de } \\
\text { categoría }\end{array}$} & \multicolumn{8}{|c|}{$\begin{array}{l}\text { Distribución de contenidos procedimentales } \\
\text { de bloques disciplinares de EGB y polimodal }\end{array}$} \\
\hline & & \multicolumn{2}{|c|}{$\begin{array}{l}\text { B1 } \\
\text { La vida y sus } \\
\text { propiedades }\end{array}$} & \multicolumn{2}{|c|}{$\underset{\text { físico }}{\text { El mundo }}$} & \multicolumn{2}{|c|}{$\begin{array}{c}\text { B3 } \\
\text { Estructura } \\
\text { y cambios } \\
\text { de la materia }\end{array}$} & \multicolumn{2}{|c|}{$\begin{array}{l}\text { B4 } \\
\text { La Tierra } \\
\text { y sus } \\
\text { cambios }\end{array}$} \\
\hline & & EGB & Pol & EGB & Pol & EGB & Pol & EGB & Pol \\
\hline $\begin{array}{l}\text { Formulación de preguntas y explicaciones } \\
\text { provisorias }\end{array}$ & $\mathrm{C} 1$ & $0 / 0 / 0$ & 0 & $0 / 1 / 0$ & 0 & $0 / 0 / 0$ & 0 & $0 / 2 / 0$ & 1 \\
\hline $\begin{array}{l}\text { Selección, recolección y organización } \\
\text { de información }\end{array}$ & $\mathrm{C} 2$ & $8 / 10 / 11$ & 3 & $12 / 13 / 12$ & 1 & $2 / 7 / 7$ & 1 & $5 / 6 / 9$ & 1 \\
\hline Interpretación de la información & $\mathrm{C} 3$ & $7 / 3 / 7$ & 4 & $3 / 10 / 6$ & 3 & $3 / 3 / 7$ & 1 & $4 / 7 / 9$ & 2 \\
\hline Diseño de investigaciones & $\mathrm{C} 4$ & $3 / 3 / 8$ & 2 & $1 / 4 / 4$ & 1 & $4 / 3 / 2$ & 2 & $1 / 4 / 2$ & 1 \\
\hline Comunicación & $\mathrm{C} 5$ & $2 / 3 / 2$ & 0 & $3 / 0 / 0$ & 0 & $2 / 2 / 2$ & 0 & $1 / 0 / 0$ & 0 \\
\hline
\end{tabular}


Tabla VIII

\begin{tabular}{|c|c|c|c|c|}
\hline \multirow{2}{*}{$\begin{array}{l}\text { Procedimientos } \\
\text { de interpretación } \\
\text { de la información }\end{array}$} & \multirow[t]{2}{*}{ CD } & \multicolumn{2}{|c|}{$\underset{\mathbf{F}}{\text { Frecuencia }}$} & \multirow{2}{*}{$\begin{array}{l}\text { Ejemplificación } \\
\text { Transcripción de contenidos procedimentales } \\
\text { de los bloques disciplinares de EGB y polimodal }\end{array}$} \\
\hline & & $\begin{array}{l}\mathrm{EGB} \\
\mathrm{N}=69\end{array}$ & $\begin{array}{c}\text { Pol } \\
\mathrm{N}=10\end{array}$ & \\
\hline Clasificación... & $\mathrm{P} 1$ & 10 & 0 & $\begin{array}{l}\text { Clasificación de frutos y semillas de acuerdo con características } \\
\text { morfológicas observables (B1, EGB1) }\end{array}$ \\
\hline Comparación... & $\mathrm{P} 2$ & 8 & 0 & $\begin{array}{l}\text { Comparación de difracción e interferencia en luz con } \\
\text { experiencias análogas en sonido (B2, EGB3) }\end{array}$ \\
\hline $\begin{array}{l}\text { Elaboración o análisis } \\
\text { de modelos } \\
\text { o explicaciones... }\end{array}$ & $\mathrm{P} 3$ & 7 & 0 & $\begin{array}{l}\text { Análisis de modelos históricos de sistema planetario } \\
\text { (B2, EGB3) }\end{array}$ \\
\hline Análisis de sistemas... & $\mathrm{P} 4$ & 2 & 0 & $\begin{array}{l}\text { Análisis de sistemas multicomponentes de la vida cotidiana: } \\
\text { salmuera, mayonesa, etc. (B3, EGB1) }\end{array}$ \\
\hline $\begin{array}{l}\text { Análisis o interpretación } \\
\text { de información... }\end{array}$ & P5 & 12 & 4 & $\begin{array}{l}\text { Interpretación de información periodística sobre fenómenos de } \\
\text { contaminación, manifestaciones volcánicas, sísmicas } \\
\text { e inundaciones (B4, EGB2) }\end{array}$ \\
\hline $\begin{array}{l}\text { Análisis o interpretación } \\
\text { de experimentos } \\
\text { o fenómenos... }\end{array}$ & P6 & 12 & 2 & $\begin{array}{l}\text { Interpretación de la caída libre de diferentes objetos } \\
\text { B2, EGB2); Análisis de resultados de experimentos originales } \\
\text { de la biología (B1, polimodal) }\end{array}$ \\
\hline Análisis de variables... & $\mathrm{P} 7$ & 2 & 1 & $\begin{array}{l}\text { Análisis de variables que interviene en el proceso de reproducción } \\
\text { de levaduras (B1, EGB2) }\end{array}$ \\
\hline Interpretación de gráficos... & $\mathrm{P} 8$ & 2 & 0 & Interpretación de gráficos de población (B1, EGB3) \\
\hline $\begin{array}{l}\text { Análisis o interpretación } \\
\text { de funcionamiento } \\
\text { o procesos... }\end{array}$ & P9 & 12 & 3 & $\begin{array}{l}\text { Análisis del funcionamiento de aparatos simples: timbre, } \\
\text { parlantes (B2, EGB3); análisis de funcionamiento de objetos } \\
\text { tecnológicos y sistemas naturales que involucran } \\
\text { fenómenos térmicos, ondulatorios, etc. (B2, polimodal) }\end{array}$ \\
\hline $\begin{array}{l}\text { Análisis o interpretación } \\
\text { de escalas... }\end{array}$ & P10 & 2 & 0 & $\begin{array}{l}\text { Interpretación de escalas de temperatura y traducción de unas } \\
\text { a otras (B2, EGB3) }\end{array}$ \\
\hline
\end{tabular}

De la lista de contenidos procedimentales, realizamos la extracción de aquéllos correspondientes a la categoría Interpretación de la información, resultando 69 contenidos en EGB y 10 en POL. Establecemos un código (CD) para los procedimientos, al solo efecto de indentificarlos en el análisis posterior.

\section{ANÁLISIS Y DISCUSIÓN}

Los contenidos de Interpretación de la información (Tablas V y VIII) se contrastan con las destrezas implicadas en las estrategias de razonamiento y de argumentación (Tabla II).

Este análisis (Tabla IX) es complejo; así, el contenido Reconocimiento de diferencias entre objetos o hechos semejantes (Tabla V, 1.a.) lo categorizamos como estrategia de razonamiento inductivo y como estrategia de argumentación, ya que, respectivamente, está relacionado con las destrezas I.3 y A.2 de la tabla II.

\section{Análisis de contenidos de Interpretación de la infor- mación del bloque 5}

Teniendo en cuenta el marco referencial (Tabla II) analizamos los contenidos procedimentales de la categoría Interpretación de la información del bloque 5 (Tabla V) y los categorizamos como estrategia de razonamiento inductivo, deductivo o de argumentación, según corresponda (Tabla IX).

Se infiere, de la tabla, el énfasis puesto en el bloque 5 en que se aprendan estrategias de razonamiento y de argumentación desde los primeros ciclos de EGB. Parece que el aprendizaje de las estrategias de razonamiento inductivo se aprende en EGB, mientras que el aprendizaje de la argumentación abarca todos los niveles. Destacamos 
Tabla IX

\begin{tabular}{|c|c|c|c|c|c|c|c|c|c|c|c|c|c|c|c|}
\hline \multirow[b]{3}{*}{ Estrategias } & \multicolumn{15}{|c|}{ Procedimientos de Interpretación de la información del bloque 5} \\
\hline & \multicolumn{9}{|c|}{ EGB } & \multicolumn{2}{|c|}{ Polimodal } & \multicolumn{4}{|c|}{$\begin{array}{l}\text { Formación de profesores } \\
\text { de ciencias }\end{array}$} \\
\hline & 1.a & $1 . b$ & 1.c & 2.a & $2 b$ & 2.c & 3.a & 3.b & 3.c & 4.a & 4.b & 5.a & $5 . \mathrm{b}$ & 5.c & 5.d \\
\hline Razonamiento inductivo & $\mathrm{x}$ & $\mathrm{x}$ & $\mathrm{x}$ & $\mathrm{x}$ & $\mathrm{x}$ & $\mathrm{x}$ & & $\mathrm{x}$ & $\mathrm{x}$ & & & & & & \\
\hline Razonamiento deductivo & & & & $\mathrm{x}$ & $\mathrm{x}$ & & & & $\mathrm{x}$ & $\mathrm{x}$ & & $\mathrm{x}$ & & & \\
\hline Argumentación & $\mathrm{x}$ & $\mathrm{x}$ & $\mathrm{x}$ & & $\mathrm{x}$ & $\mathrm{x}$ & $\mathrm{x}$ & $\mathrm{x}$ & $\mathrm{x}$ & $\mathrm{x}$ & $\mathrm{x}$ & $\mathrm{x}$ & $\mathrm{x}$ & $\mathrm{x}$ & $\mathrm{x}$ \\
\hline
\end{tabular}

la relevancia del papel de la argumentación en la FPC y la forma gradual que se propone para su aprendizaje.

\section{Análisis de contenidos de interpretación de la infor- mación de los bloques disciplinares}

Recordamos que el rol de la comunicación en los bloques disciplinares no implica destrezas de argumentación, se limita a la comunicación de resultados y no hay lugar para las prácticas discursivas (Driver y Newton, 2000).

Centramos el análisis en el contraste de los procedimientos (Tabla VIII) con las estrategias de razonamiento inductivo y deductivo (Tabla II), estableciéndose la categorización que se recoge en la tabla $\mathrm{X}$.

Hemos de destacar que los diseñadores de los bloques disciplinares del nuevo currículo de EGB y POL proponen el aprendizaje de las disciplinas del área de ciencias utilizando, casi exclusivamente, estrategias de razonamiento inductivo.

\section{Discusión de resultados}

La comparación de los resultados recogidos en las tablas IX y X pone de manifiesto los diferentes puntos de vista, respecto al razonamiento y a la argumentación, que coexisten en el nuevo currículo de ciencias de la reforma educativa argentina. Mientras los especialistas que diseñaron el bloque específico de procedimientos proponen aprender a razonar tanto individual como colectivamente y tienen en cuenta el aprendizaje gradual de los razonamientos con visión propedéutica, los especialistas que redactan los contenidos de las disciplinas parecen considerar que el conocimiento de la ciencia escolar se aprende razonando individual e inductivamente.

Los especialistas que redactan el bloque 5 fomentan el aprendizaje de nuevas destrezas, especialmente las implicadas en las estrategias de argumentación; en cambio, los especialistas que redactan los bloques disciplinares proponen contenidos procedimentales que reflejan prácticas basadas en visiones positivistas.

Llama la atención el bajo número de destrezas incluidas en las estrategias de razonamiento deductivo, tanto en el bloque 5 como en los disciplinares. Esto podría indicar poco interés por enseñar a razonar a partir de hipótesis y modelos. Consideramos que la ausencia en las clases de ciencias del aprendizaje del razonamiento deductivo implicaría no dar oportunidades a los estudiantes para el desarrollo de ciertas destrezas necesarias a la hora de tomar decisiones en base a argumentos, como se solicita en polimodal.

Es relevante la diferencia en la forma de redactar los contenidos en las partes analizadas. Mientras en el

Tabla X

\begin{tabular}{|l|c|c|c|c|c|c|c|c|c|c|}
\cline { 2 - 10 } & \multicolumn{8}{c|}{ Procedimientos de Interpretación de la información de los bloques disciplinares de EGB y polimodal } \\
\hline Estrategias & $\mathrm{P} 1$ & $\mathrm{P} 2$ & $\mathrm{P} 3$ & $\mathrm{P} 4$ & $\mathrm{P} 5 *$ & $\mathrm{P} 6 *$ & $\mathrm{P} 7 *$ & $\mathrm{P} 8$ & $\mathrm{P} 9 *$ & $\mathrm{P} 10$ \\
\hline Razonamiento inductivo & $\mathrm{x}$ & $\mathrm{x}$ & $\mathrm{x}$ & $\mathrm{x}$ & $\mathrm{x}$ & $\mathrm{x}$ & $\mathrm{x}$ & $\mathrm{x}$ & $\mathrm{x}$ & $\mathrm{x}$ \\
\hline Razonamiento deductivo & & & & & & & & & $\mathrm{x}$ & \\
\hline Argumentación & & & & & & & & & & \\
\hline \multicolumn{1}{|c|}{} \\
\hline
\end{tabular}


bloque 5 se evidencia una redacción pedagógica de lo que se debe saber hacer (Tabla V), en los bloques disciplinares simplemente se enuncian operaciones cognitivas aplicadas sobre un contenido conceptual (Tabla VIII). Por otra parte, en los bloques disciplinares, una misma operación cognitiva (p.e., análisis) se solicita en distintos ciclos de EGB, sin que surja con qué profundidad se debe aprender en cada uno. En cambio, en el bloque 5, los contenidos procedimentales hacen explícita la gradación en el aprendizaje (p.e. 1.a;2.a;3.a, Tabla $\mathrm{V})$ de modo tal que no hay repetición, sino un camino de progreso hacia su logro (p.e., 4.a, Tabla V).

No se encuentra, en los bloques disciplinares de EGB, ningún contenido procedimental que se corresponda con el contenido 3.a (Tabla V), el cual, según interpretamos, expresa el nivel de argumentación con que un alumno debería egresar del nivel EGB. Esto constituye un obstáculo para el aprendizaje gradual de prácticas discursivas que, según surge del bloque 5 , supone muchos años de ciencia escolar para su logro y deberían estar aprendidos al egresar de POL.

\section{CONCLUSIONES E IMPLICACIONES}

\section{Sobre el tema investigado}

Aprender a argumentar en ciencias es un propósito explícito por el que se incorporan los contenidos procedimentales en el currículo y, sin duda, guía la redacción del bloque 5, específico de los Procedimientos. Sin embargo, este estudio muestra que los bloques disciplinares del mismo currículo no lo tienen en cuenta.

Los contenidos procedimentales del nuevo currículo de ciencias de la reforma argentina reflejan la práctica de visiones epistemológicas diferentes. Mientras los redactores del bloque 5 proponen contenidos que fomentan el aprendizaje de estrategias de razonamiento y de argumentación en todos los niveles de enseñanza investigados, los especialistas de las disciplinas proponen prácticas de razonamiento inductivo y olvidan la creación de contextos propicios para la argumentación.

La falta de relación entre los contenidos procedimentales de las distintas partes de un mismo currículo (Tablas V y VIII), que según fuentes oficiales se elaboró a partir de discusiones entre especialistas, parecería mostrar que los contenidos del bloque 5 o bien han sido ignorados, o bien no ha sido posible su transposición por los especialistas. Parece que el discurso pedagógico y el discurso de la disciplina coexisten en un mismo currículo de ciencias sin que existan acuerdos sobre los procedimientos de la ciencia escolar.

El aprendizaje de las ciencias es propuesto por los especialistas como un proceso callado e individual y el razonamiento se concibe como una construcción individual organizada sobre un patrón de razonamiento inductivo. Resulta relevante conocer esta visión de los redactores de las disciplinas del currículo, quienes proponen aprender ciencias casi sin hablar ciencias, puesto que esta visión puede constituir criterio para el profesorado de ciencias a la hora de diseñar su proyecto de aula. No debería olvidarse que sin un contexto de negociación social y de cambio actitudinal (Duschl, 1995), el alumnado no tendrá oportunidad para realizar prácticas discursivas que fomenten la argumentación.

El término discurso tiene diferentes significados en función del soporte en el que se hace explícito y del escenario en el que se produce: puede ser escrito u oral e individual o colectivo. Esto no es de extrañar, pues en la propia área de didáctica de las ciencias se está produciendo esta polisemia (Driver y Newton, 2000; Jiménez, 2001; Yore, 2001).

\section{Sobre el instrumento utilizado}

En este estudio, el marco referencial (Tabla II) se constituyó en una herramienta útil, ya que permitió evaluar los contenidos procedimentales respecto al razonamiento y a la argumentación. Durante el proceso de evaluación de las destrezas implicadas en la argumentación, cumplió un papel relevante, el campo social y de comunicación (Duschl, 1995), a través del cual se reflejaron visiones epistemológicas que fomentaron o no las prácticas discursivas en el aula de ciencias.

El proceso de vaciado de los contenidos procedimentales facilitó la extracción y la categorización de contenidos que implican destrezas de razonamiento y de argumentación.

\section{Implicaciones en la enseñanza}

Constituyendo el currículo oficial material de referencia para instituciones y docentes, destacamos la problemática que representa encontrar dos visiones diferentes sobre el razonamiento científico. De no existir una reflexión profunda sobre la cuestión, para el profesorado será más económico, intelectual y profesional utilizar la visión positivista, dado que la visión constructivista implicaría no sólo cambio y ruptura epistemológica, sino además que el profesorado aprenda y enseñe estrategias intelectuales que tradicionalmente se consideraron como de evolución natural.

En el currículo de formación de profesores de ciencias, parece que se consideran adquiridas las estrategias señaladas en el currículo de EGB y de POL. Dado que la reforma educativa argentina es reciente, consideramos conveniente que las carreras de profesorado potencien el aprendizaje del razonamiento y de la argumentación sin el supuesto previo, posiblemente no acertado, que deberían ser estrategias ya adquiridas. Implica un desafío para el profesorado de las didácticas específicas, colaborar para que en el aula de ciencias sea posible la integración entre los contenidos procedimentales enunciados en el bloque 5 y los contenidos conceptuales de las disciplinas. Debemos tener en cuenta que el conocimiento conceptual específico es un factor determinante 
en la eficacia para el uso de estrategias de razonamiento y de argumentación (Álvarez et al., 1998; Jiménez, 1998) y que éstas no se aprenden con independencia del contenido al que se aplican (Pérez y Pozo, 1994; Couce et al., 1998).

En tiempo de reforma educativa y frente al excesivo énfasis que los currículos oficiales ponen en los contenidos conceptuales, en particular en lo concerniente a los bloques disciplinares, se debería tener en cuenta la necesidad de integrarlos con los contenidos procedimentales y actitudinales en el proceso de enseñanza y aprendizaje de las ciencias. Señala el marco legal (MCE, 1997b, p. 147) que «las estrategias de investigación y

\section{REFERENCIAS BIBLIOGRÁFICAS}

ÁLVAREZ, V.M., BERNAL, M. y GARCÍA-RODEJA, E. (1998). Destrezas argumentativas en física: un estudio de caso utilizando problemas sobre flotación, en Banet, E. y Pro, A. (coords.). Investigación e Innovación en la enseñanza de las ciencias, pp. 30-42. Lleida: DM.

ANDERSON, J.R.(1983). The architecture of cognition. Londres: Harvard Universty Press.

CAMPANARIO, J.M. y OTERO, J.C. (2000). Más allá de las ideas previas como dificultades de aprendizaje: las pautas de pensamiento, las concepciones epistemológicas y las estrategias metacognitivas de los alumnos de ciencias. Enseñanza de las Ciencias, 18(2), pp. 155-169.

CLAXTON, G. (1991). Educating the inquiring mind. The challenge for school science. Londres: Harvester. Trad. cast. (1994). Educar mentes curiosas. El reto de la ciencia en la escuela. Madrid: Visor.

COUCE, A., DOMíNGUEZ, J.M. y ÁLVAREZ, V. (1998). Argumentación del alumnado de $2^{\circ}$ de ESO sobre un problema relacionado con la formación de sombras, en Martínez Losada, C. y García Barros, S. (eds.). La didáctica de las ciencias. Tendencias actuales. Coruña: Servicio de Publicaciones de la Universidad de la Coruña.

DGCE (1995). Ley Federal de Educación. Ley de Educación de la Pcia. de Buenos Aires. Contenidos Básicos Comunes. Módulo O. Gobierno de la Provincia de Buenos Aires. Capítulo de Ciencias Naturales. La Plata. Argentina, pp. 167- 175.

DGCE (1998). Definiciones para el diseño del proyecto institucional curricular, p. 13. Dirección de Educación Media, Técnica y Agraria. Programa de Transformación Educativa de la Provincia de Buenos Aires. La Plata. Argentina.

DGCE (1999a). Diseño curricular. Educación inicial. Educación general básica. Tomo II. Resolución núm. 13.227/99.

DGCE (1999b). Fundamentación de la estructura curricular de la formación docente 3 r. ciclo de la EGB y nivel polimodal, p. 32. los procedimientos implicados en ellas son contenidos que suelen estar prácticamente ausentes en la formación docente, y esta carencia resulta un obstáculo serio pues los docentes no pueden enseñar aquello que no han aprendido».

\section{AGRADECIMIENTOS}

A la UN del Centro. Olavarría (Argentina) y a la USC (España), el convenio de cooperación conjunta.

A la DGES, la financiación del proyecto (PB98-0616).

DOMÍNGUEZ, J.M. (2000). «Evolución de las formas de hacer y de pensar sobre un sistema material, en el marco de la termodinámica y del modelo de partículas. Estudio mediante esquemas de acción y de razonamiento». Tesis doctoral. Servicio de Publicaciones de la Universidad de Santiago de Compostela. Santiago de Compostela.

DOMÍNGUEZ, J.M., PRO, A, GARCÍA-RODEJA, E. y ILLOBRE, M. L. (1999). Os esquemas de acción e a avaliación do coñecemento procedimental. A medida da temperatura na ESO. ADAXE. Revista de Estudios e Experiencias Educativas, 14-15, pp. 233-270.

DRIVER, R. y NEWTON, P. (2000). Establishing the norms of scientific argumentation in classrooms. Science Education, 84(3), pp. 287-312.

DUSCHL, R.A. (1990). Reestructuring Science Education. Nueva York: Teachers College Press. Trad. de Álvarez, V. (1997). Renovar la enseñanza de las ciencias. Importancia de las teorías y su desarrollo. Madrid: Narcea.

DUSCHL, R.A. (1995). Mas allá del conocimiento: los desafíos epistemológicos y sociales de la enseñanza mediante el cambio conceptual. Enseñanza de las Ciencias, 13(1), pp. 3-14.

EICHINGER, D., ANDESSON, C.W., PALINCSAR, A.S. y DAVID, Y.M. (1991). An illustration of the roles of Content Knowledge, Scientific Argument and Social norms in Collaborative Problem-solving. Documento presentado a la conferencia anual de AERA. Chicago.

GIERE, R.N. (1994). The cognitive structure of scientific theories. Philosophy of Science, 61, pp. 276-296.

GIERE, R.N. (1999). Un nuevo marco para enseñar el razonamiento científico. Enseñanza de las Ciencias, núm. extra, pp. 63-70.

HEAD, J. (1985). The Personal Response to Science. Londres: Cambridge University Press.

IZQUIERDO, M. (2000). Hablar y escribir para aprender. Actas de los XIX Encuentros de Didáctica de las Ciencias Experimentales, pp. 17-29. Madrid. 
JIMÉNEZ, M.P. (2001). Discurso de aula y argumentación en la clase de ciencias. Enseñanza de las Ciencias, núm. extra. VI Congreso, pp. 61-62.

JIMÉNEZ, M.P., BUGALLO, A. y DUSCHL, R.A. (1997). Argument in High Scholl Genetics. Documento presentado en la conferencia anual de la NARST. Chicago.

JIMÉNEZ, M.P. (1998). Diseño curricular: indagación y razonamiento con el lenguaje de las ciencias. Enseñanza de las Ciencias, 12(3), pp. 299-313.

JIMÉNEZ, M.P., GARCÍA-RODEJA, I., ÁLVAREZ, V., LAGO, J.M. y RIAL, R. (1998). La argumentación en los libros de texto de ciencias. Ponencia presentada en el Seminario «Comprensión y producción de textos científicos». Aveiro, Portugal.

JORBA, J. y SANMARTÍ, N. (1996). El desarrollo de las habilidades cognitivo-lingüísticas en la enseñanza científica. Seminario Desplegament curricular. ICE de la UAB. Septiembre 1996. Barcelona.

KELLY, G.J., DRUCKER, S. y CHEN, K. (1996). Students' reasoning about electricity: combining performance assessment with argumentation analysis. Documento presentado en la Conferencia anual de AERA. Nueva York.

KESIDOU, S. y DUIT, R. (1993). Student's Conceptions of the Second Law of Thermodynamics - An Interpretative Study. Journal of Research in Science Teaching, 30(1), pp. 85-106.

KUHN, D. (1993). Science as argument: Implications for teaching and learning scientific thinking. Science Education, 77(3), pp. 319-337.

LATOUR, B. y WOOLGAR, S. (1995). La vida en el laboratorio: la construcción de los hechos científicos. Madrid: Alianza.

LEMKE, J. L. (1993). Talking science: language, learning and values. Ablex. Trad. de García, A. et al. (1997). Aprender a hablarde ciencia: Lenguaje, aprendizaje y valores. Barcelona: Paidós.

MCE (1995). Contenidos Básicos para la Educación General Básica. República Argentina, pp. 113-168.

MCE (1996). Los CBC en la escuela . Primer ciclo. Ministerio de Cultura y Educación de la Nación. República Argentina.

MCE (1997a). Contenidos básicos para la educación polimodal, pp. 11-137 y 263-285. República Argentina.

MCE (1997b). Contenidos básicos comunes del área de ciencia naturales en la formación docente de grado para nivel inicial y primer y segundo ciclo de EGB, en Contenidos básicos comunes para la formación docente de grado, pp. 139-160. República Argentina.

MCE (1997c). Materiales de apoyo para la capacitación docente: EGB. Caracterización de los capítulos de los CBC, pp. 127130. República Argentina.

MCE (1998). Contenidos básicos comunes del campo de la formación docente (Formación disciplinar específica: Profesor de Física para Tercer Ciclo de EGB y Polimodal). Materiales de trabajo de los Contenidos Curriculares Básicos para la Formación Docente. Resolución 74/98.
MILLAR, R.H. y DRIVER, R. (1987). Beyond Processes. Studies in Science Education, 14, pp. 33-62.

MINICK, C. y ALVERMANN, D.E. (1994). Una didáctica de las ciencias. Procesos y aplicaciones. Buenos Aires: Aique.

MONEREO, C. y CASTELLÓ, M. (1997). Las estrategias de aprendizaje. Cómo incorporarlas a la práctica educativa. Barcelona: EDEBE.

PÉREZ, M.P. y POZO, J.I. (1994). Aprender a resolver problemas y resolver problemas para aprender, en Pozo, J.I. (ed.) Solución de problemas. Madrid: Santillana.

PONTECORVO, C. y GIRARDET, H. (1993). Arguing and reasoning in Understanding Historical Topics. Cognition and Instruction, 11(3 y 4), pp. 365-395.

POZO, J.I. (1996). Aprendices y maestros. Madrid: Alianza/ Psicología Minor.

POZO, J.I., POSTIGO, Y. y GÓMEZ, M.A. (1995). Aprendizaje de estrategias para la solución de problemas en ciencias. Alambique, 5, pp. 16-26.

POZO, J.I. y GÓMEZ, M.A. (1998). Aprender y enseñar ciencia. Del conocimiento cotidiano al conocimiento científico. Madrid: Morata.

PRO, A. (1995). Reflexiones para la selección de contenidos procedimentales en ciencias. Alambique, 6, pp. 77-87.

PRO, A. (1998) ¿Se pueden enseñar contenidos procedimentales en las clases de ciencias? Enseñanza de las Ciencias, 16(1), pp. 21-41.

SANMARTÍ, N. (1997). Enseñar a elaborar textos científicos en las clases de ciencias. Alambique, 12, pp. 51-61.

SARDÁ, A y SANMARTÍ, N. (2000). Enseñar a argumentar científicamente: un reto de las clases de ciencias. Enseñanza de las Ciencias, 18(3), pp. 405-422.

SEVILLA, C. (1994). Los procedimientos en el aprendizaje de la física. Enseñanza de las Ciencias, 12(3), pp. 400-405.

TISHMAN, S., PERKINS, D. y JAY, E. (1994). The thinking classroom and teaching in a culture of thinking. Allyn and Bacon. Trad. de Wald, M. (1994). Un aula para pensar. Buenos Aires: Aique.

VAN HIELE, P.M. (1986). Structure and insight. A theory of mathematics education. Academic Press: Londres, en Jaime Pastor, A. y Gutiérrez Rodríguez, A. (1996). El grupo de las isometrías del plano. Madrid: Síntesis.

VERGNAUD, G. (1994). Apprentissages et didactiques, ou en est-on? Hacchette Livre. Trad. de Maranzano, C. (1997). Aprendizajes y didácticas. ¿Qué hay de nuevo? Buenos Aires: Edicial.

YORE, L.D. (2001). Authentic Practices in Science Discourse Communities: Implications for Language Arts Science Education. Documento presentado a la Conference ScienceLiteracy at the University of Maryland. Baltimore County, Baltimore, MD. Agosto. 